\title{
The Influence of Sampling Methods on Pixel-Wise Hyperspectral Image Classification with 3D Convolutional Neural Networks
}

\author{
Julius Lange ${ }^{1}$, Gabriele Cavallaro ${ }^{2}$, Markus Götz| ${ }^{2,3}$, Ernir Erlingsson $^{3}$, Morris Riedel ${ }^{2,3}$ \\ ${ }^{1}$ Humboldt University of Berlin \\ ${ }^{2}$ Jülich Supercomputing Centre, Forschungszentrum Jülich \\ ${ }^{3}$ School of Engineering and Natural Sciences, University of Iceland
}




\section{Outline}

Introduction

Proposed Cluster Sampling

Experimental Results

Conclusions 


\title{
Outline
}

\author{
Introduction
}

Proposed Cluster Sampling

Experimental Results

Conclusions 


\section{Remote Sensing Image Classification}

\section{Supervised Learning}

- Annotated samples are available

- Supervised optimization of the classifier parameters

- How to obtain reliable semantic maps from unseen data?
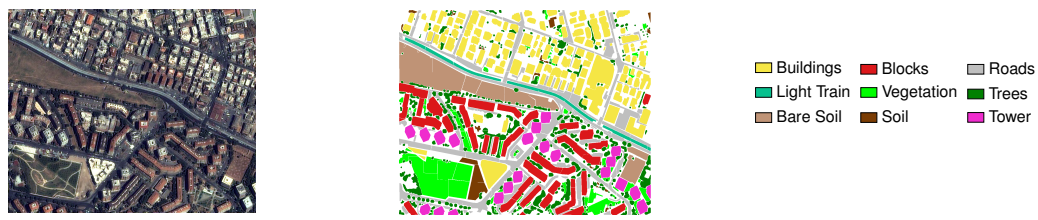


\section{Spectral-Spatial Classifiers}

The Value of Spatial Information

- Spectral classifiers: can't deal with high spatial resolution images

- Exploit the spatial autocorrelation of data

- Improving the understanding of remote sensing images

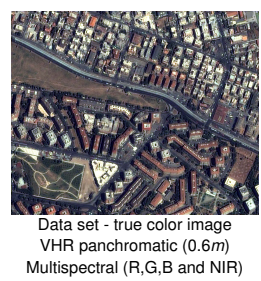

August 21, 2018

Multispectral (R,G,B and NIR)

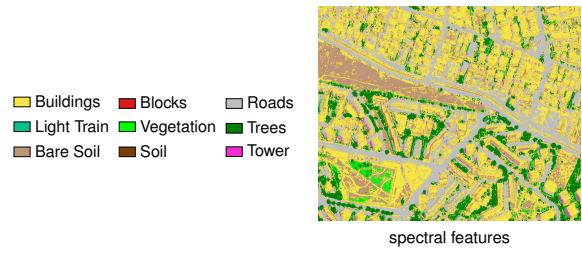

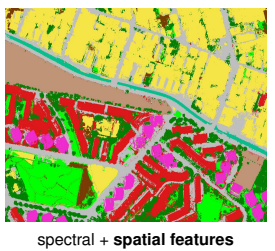

spectral + spatial features 


\section{Spatial Autocorrelation}

Remote Sensing Data

- Spectral dependence degree between a pixel and its neighbors

- Measure of statistical separability between spatial objects

1 Intrinsic property $\rightarrow$ types of land cover classes

2 Spatial resolution $\rightarrow$ pixel's size

3 Pre-processing $\rightarrow$ feature engineering, CNNs, etc.

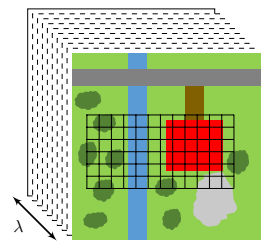

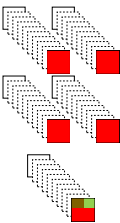

Thematic classes:

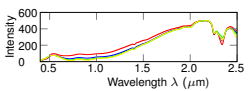

$\square$ Roof $\square$ Grass $\square$ Tree $\square$ Bare Soil $\square$ Soil $\square$ Road $\square$ Water

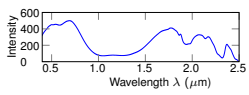




\section{Accuracy Assessment}

\section{Sampling Step}

- Influenced by:

- The model of the classifier

- The sampling scheme

- Groundtruth split into three disjoint sets: training, validation and test

- Sampler determines amount and distribution of samples across the scene

- It can significantly affect the test phase

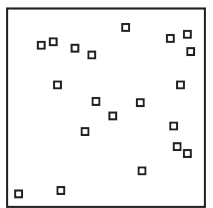

Random sampling

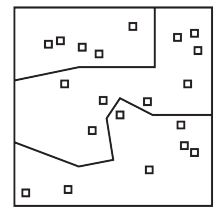

Stratified random sampling

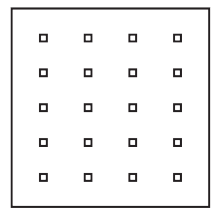

Systematic sampling

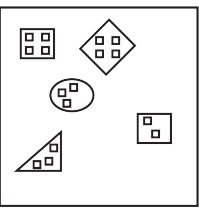

Cluster sampling 


\section{Motivation}

What this Work is About

- Practice of training and validating new classifiers within a single image

- Groundtruth split with random sampling

- It was a natural choice for spectral classifier

- Could already violate the independence assumption (bias train and test sets)

- Inevitable spatial autocorrelation between adjacent pixels

- Direct neighboring or nearby pixels present in both train and test sets

- Spatial closeness: information from one set may leak into the respective other 


\section{Motivation}

What this Work is not About

- Existing datasets have a number of limitations

- Lack of image variations and diversity

- Saturation of accuracy

\begin{tabular}{|c|c|}
\hline Dataset and Reference & Number of uses \\
\hline IEEE GRSS 2013 Data Fusion Contest ${ }^{338}$ & 4 \\
\hline IEEE GRSS 2015 Data Fusion Contest ${ }^{339}$ & 1 \\
\hline IEEE GRSS 2016 Data Fusion Contest ${ }^{340}$ & 2 \\
\hline Indian Pines $^{341}$ & 27 \\
\hline Kennedy Space Center ${ }^{342}$ & 8 \\
\hline Pavia City Center ${ }^{343}$ & 13 \\
\hline Pavia University ${ }^{343}$ & 19 \\
\hline Salinas $^{344}$ & 11 \\
\hline Washington DC Mall ${ }^{345}$ & 2 \\
\hline
\end{tabular}

Open-source Hyperspectral datasets used for DL papers, John Ball, 2017 [1] 


\section{Motivation}

What this Work is not About

- Transfer learning, domain adaptation and active learning

- Recent advancement in EO benchmark data creation

\begin{tabular}{|c|c|c|c|c|c|c|c|}
\hline Dataset & Image per class & Scene classes & Total images & Spatial resolution $(\mathrm{m})$ & Image sizes & Year & Refernce \\
\hline UC Merced Land-Use & 100 & 21 & 2100 & 0.3 & $256 \times 256$ & 2010 & [2] \\
\hline WHU-RS19 & 50 & 19 & 1005 & up to 0.5 & $600 \times 600$ & 2012 & [3] \\
\hline RSSCN7 & 400 & 7 & 2800 & - & $400 \times 400$ & 2015 & [4] \\
\hline SAT-6 & - & 6 & 405000 & 1 & $28 \times 28$ & 2015 & [5] \\
\hline Brazilian Coffee Scene & 1438 & 2 & 2876 & - & $64 \times 64$ & 2016 & [6] \\
\hline SIRI-WHU & 200 & 12 & 2400 & 2 & $200 \times 200$ & 2016 & [7] \\
\hline NWPU-RESISC45 & 700 & 45 & 31500 & 30 to 0.2 & $256 \times 256$ & 2016 & [8] \\
\hline AID & 300 & 30 & 10000 & 0.6 & $600 \times 600$ & 2017 & [9] \\
\hline EuroSAT & 2500 & 10 & 27000 & 10 & $64 \times 64$ & 2017 & [10] \\
\hline RSI-CB128 & 36000 & - & 45 & 3 & $128 \times 128$ & 2017 & [11] \\
\hline RSI-CB256 & 24000 & - & 35 & 0.3 & $256 \times 256$ & 2017 & [11] \\
\hline
\end{tabular}

- Yet not enough (e.g., Imagenet with 14,197,122 images)

- Data variation between train and application phase remains in place 


\section{Outline}

\section{Introduction}

Proposed Cluster Sampling

\section{Experimental Results}

\section{Conclusions}




\section{Aims of the Approach}

- Capture the full spectral variation of the image

- Reduces overlap between train and test samples due to spatial processing

1 Extract larger contiguous regions using the class labels

2 Distribute them disjointly between the train and test set

- A bias, if present at all, would then only be relevant at the outer edges of such a region, but not for the inner pixels

- More objective and accurate evaluation 


\section{DBSCAN}

Density-Based Spatial Clustering for Applications with Noise

- Can be used for computing n-connected components in an image

- They can have gaps ( $\varepsilon$-sizes), filtered by (minPoints-area)

- Able to detect arbitrarily shaped clusters

- Don't need to know the number of clusters a priori

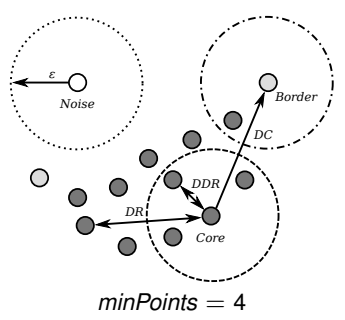

- Cluster core: point that contains within a spatial search radius $\varepsilon$ at least a certain number of neighboring points minPoints 


\section{Extraction of Contiguous Regions}

- Cluster the coordinates of pixels of each of the individual classes

- Each cluster corresponds to one of the identified region of that class

- DBSCAN performs n-connected-component-labeling

- $\varepsilon=\{1, \sqrt{2}\}$ for 4- and 8-connectivity, respectively

- minPoints serves as threshold for potential gaps

- i.e. If minPoints $<$ num. of connected components $+1 \rightarrow$ increasingly larger gaps 


\section{Regions Distribution Between the Train and Test Set}

\section{Not Efficient Approaches}

- Regions could be randomly assigned to either one of the two sets

- However, num. of regions $<<$ num. of pixels within each region

- The likelihood of selecting an imbalanced train set rises

- The set might not contain patterns present in the test set

- Each region could be subdivided into two disjoint parts

- E.g., the "top" and "bottom"

- However, the number of biased pixels increases along the partition boundary 


\section{Regions Distribution Between the Train and Test Set}

\section{Proposed Approach}

- metric function assigns to all regions of a class a partially ordered ranking

- It indicates their assignment priority to the train or test set

- Regions are added to a set until the pixels class split fraction is reached

- The region causing an over pixels assignment is split into two sub-regions

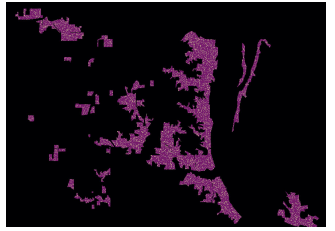

Random sampling

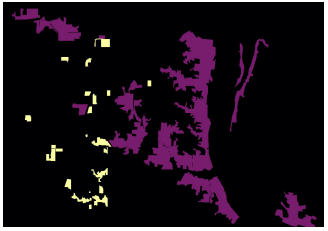

Cluster sampling (area)

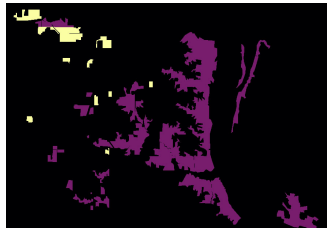

Cluster sampling (StdDev) 


\title{
Outline
}

\section{Introduction}

\section{Proposed Cluster Sampling}

\author{
Experimental Results
}

\section{Conclusions}




\section{Dataset}

\section{University of Houston - 2013 GRSS data fusion contest}

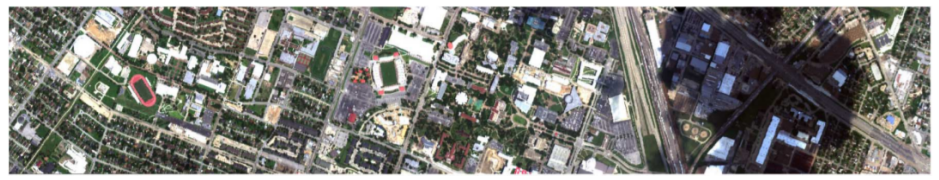

Hyperspectral image (144 spectral bands with $2.5 \mathrm{~m}$ spatial resolution)

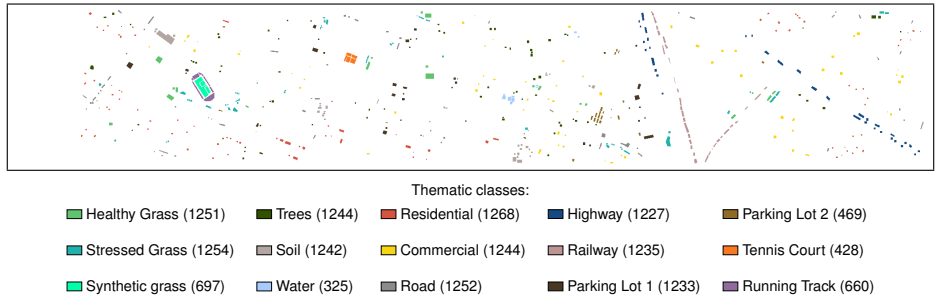




\section{Dataset}

\section{Indian Pines - AVIRIS - NASA (1992)}

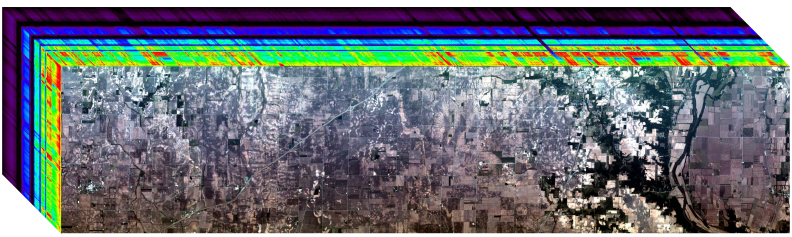

Hyperspectral image (220 spectral bands with $20 \mathrm{~m}$ spatial resolution)

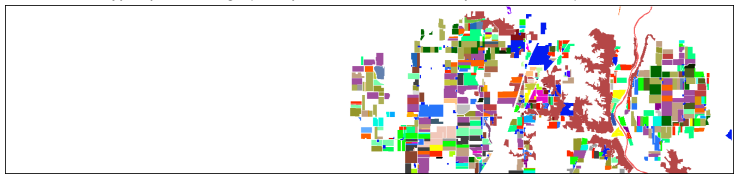

$\begin{array}{ll}\square \text { BareSoil (57) } & \square \text { Corn-MinTill (1049) } \\ \square \text { Soybeans-CleanTill (5074) } & \square \text { Soybeans-NaTill-EW (2533) } \\ \square \text { Hay-Alfalfa (2258) } & \square \text { Soybeans-CleanTill (pre.) } \\ \square \text { Soybeans-CleanTill-EW (11802) } & \square \text { Soybeans-NaTill-Drilled (873) } \\ \square \text { Swampy Area (583) } & \square \text { Corn-EW (514) } \\ \square \text { Corn-NS (2356) } & \square \text { Fescue (114) } \\ \square \text { Grass (1147) } & \square \text { Orchard (39) } \\ \square \text { Pasture (10386) } & \square \text { Soybeans-Drilled (2667) } \\ \square \text { Soybeans-MinTill (1832) } & \text { Unknown (144) } \\ \square \text { Corn-CleanTill-NS (pre.) (1728) } & \square \text { Grass-runway (37) }\end{array}$

August 21, 2018

\begin{tabular}{|c|c|}
\hline Hay (1128) & Soybeans-NS (1110) \\
\hline 口Hay (pre.) (2185) & 口Corn-MinTill-EW (5629) \\
\hline 口 Soybeans-NaTill-NS (929) & 口Corn (17783) \\
\hline$\square$ Corn (presumed) (158) & $\square$ Corn-Natiil-EW (1206) \\
\hline 口Corn-Natill-NS (5685) & 口Oats (1742) \\
\hline 口Oats (pre.) (335) & $\square$ Soybeans-CleanTill-Weedy \\
\hline 口 Soybeans-MinTill (15118) & $\square$ Wheat (4979) \\
\hline 口Woods (63562) & $\square$ Corn-CleanTill-NS (39678) \\
\hline 口Corn-CleanTill-NS-Irrigated (800) & 口Grass/Pasture (73) \\
\hline
\end{tabular}

口Soybeans (pre.) (894)

\begin{abstract}
口Soybeans-NaTill (2157)
$\square$ Concrete/Asphalt (69)

口Corn-Natill (4381)

$\square$ NotCropped (1940)

$\square$ Soybeans-CleanTill-Drilled (2242)

$\square$ Trees (pre.) (580)

口Corn-CleanTill-EW (26486)

- Grass/Pasture-mowed (19)

口Soybeans (9391)

DBuildings (17195)

$\square$ Corn-MinTill-NS (8862)

口Lake (224)

$\square$ Soybeans-CleanTill-NS (10387)

$\square$ River (3110)

$\square$ Corn-CleanTill (12404)

GGrass/Trees (2331)

$\square$ Pond (102)

口Soybeans-MinTril-Drilled (8098)
\end{abstract}

g.cavallaro@fz-juelich.de 


\section{Experimental Setup (1)}

Feature Engineering Steps Combined with Support Vector Machine

- Data dimensionality reduction

- Kernel Principal Component Analysis (KPCA)

- Spatial information enhancement

- Extended Self-Dual Attribute Profiles (ESDAPs)

- Feature extraction

- Nonparametric Weighted Feature Extraction (NWFE)

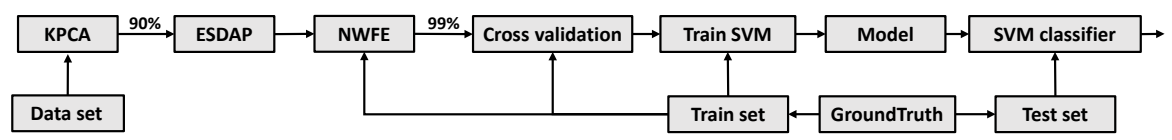




\section{Experimental Setup (2)}

\section{D Convolutional Neural Network}

\begin{tabular}{|l|l|}
\hline Feature & Representation / Value \\
\hline \hline Conv. Layer Filters & $48,32,32$ \\
Conv. Layer Filter size & $(3,3,5),(3,3,5),(3,3,5)$ \\
Pooling size & $(1,1,3),(1,1,3),(1,1,2)$ \\
Dense Layer Neurons & 128,128 \\
Activation Functions & rectified linear unit (ReLU) \\
Loss Function & mean-squared error (MSE) \\
Optimization & stochastic gradient descent (SGD) \\
Training Epochs & 600 \\
Batch Size & 50 \\
Learning Rate & 1.0 \\
Learning Rate Decay & $5 \times 10^{-6}$ \\
\hline
\end{tabular}

$\begin{array}{ccccc}\begin{array}{l}\text { Input: } \\ \text { Window Tensor }\end{array} \quad \text { 3D Convolution } & \begin{array}{c}\text { 1D Max Pooling } \\ \text { (spectral dimension) }\end{array}\end{array}$ Flatten $\quad \begin{gathered}\text { Fully Connected } \\ \text { Layers }\end{gathered} \begin{gathered}\text { Softmax } \\ \text { Layer }\end{gathered} \begin{gathered}\text { Output: } \\ \text { Probabilities }\end{gathered}$
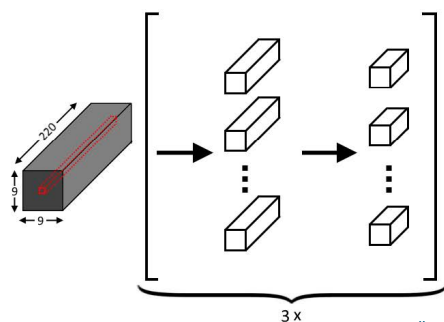
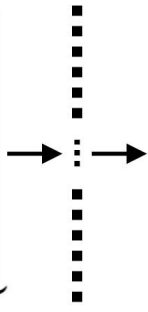

Layers

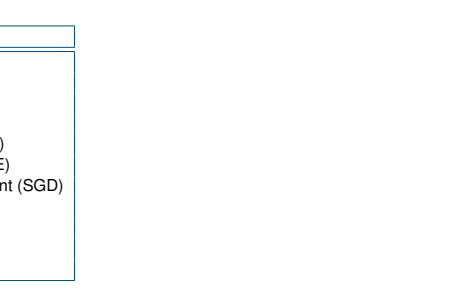




\section{Experimental Results}

\section{University of Houston}

\begin{tabular}{|c|c|c|c|c|c|c|c|c|c|}
\hline \multirow{3}{*}{$\begin{array}{l}\text { Sampling } \\
\text { method }\end{array}$} & \multicolumn{4}{|c|}{ Feature Engineering with SVM } & \multicolumn{4}{|c|}{ 3DCNN } & \multirow{3}{*}{ Metric } \\
\hline & \multicolumn{8}{|c|}{ Training set size (\%) } & \\
\hline & 10 & 30 & 60 & 90 & 10 & 30 & 60 & 90 & \\
\hline \multirow{4}{*}{ Random } & $97.68(0.09)$ & $99.62(0.03)$ & $99.85(0.04)$ & $99.90(0.07)$ & $96.03(0.42)$ & $98.97(0.13)$ & $99.51(0.23)$ & $99.75(0.14)$ & OA \\
\hline & $97.73(0.05)$ & $99.63(0.02)$ & $99.87(0.04)$ & $99.96(0.06)$ & $95.26(0.49)$ & $98.86(0.12)$ & $99.40(0.37)$ & $99.73(0.24)$ & AA \\
\hline & $97.54(0.06)$ & $99.59(0.02)$ & $99.84(0.04)$ & $99.89(0.08)$ & $95.70(0.46)$ & $98.89(0.14)$ & $99.47(0.25)$ & $99.73(0.16)$ & Kappa \\
\hline & $97.76(0.14)$ & $99.61(0.03)$ & $99.83(0.04)$ & $99.91(0.07)$ & $95.60(0.45)$ & $98.93(0.13)$ & $98.20(2.75)$ & $99.74(0.19)$ & $\mathrm{F} 1$ \\
\hline \multirow{4}{*}{$\begin{array}{c}\text { Size } \\
\varepsilon=\sqrt{2} \\
\text { minPoints }=9\end{array}$} & 50.15 & 69.49 & 79.19 & 82.89 & $53.90(2.86)$ & $75.50(0.66)$ & $83.87(1.33)$ & $87.06(0.51)$ & $\mathrm{OA}$ \\
\hline & 50.15 & 69.50 & 79.21 & 82.94 & $59.39(3.88)$ & $76.59(0.59)$ & $82.95(1.13)$ & $86.55(1.57)$ & AA \\
\hline & 46.22 & 67.07 & 77.52 & 81.48 & $50.41(3.07)$ & $73.50(0.71)$ & $82.55(1.44)$ & $86.01(0.56)$ & Kappa \\
\hline & 54.24 & 70.94 & 78.95 & 80.67 & $53.84(4.05)$ & $77.18(1.03)$ & $82.48(1.96)$ & $85.60(1.39)$ & $\mathrm{F} 1$ \\
\hline \multirow{4}{*}{$\begin{array}{c}\text { StdDev } \\
\varepsilon=\sqrt{2} \\
\text { minPoints }=9\end{array}$} & 63.47 & 66.62 & 74.01 & 80.36 & $58.50(0.71)$ & $58.37(0.69)$ & $70.59(0.47)$ & $79.36(2.07)$ & $O A$ \\
\hline & 63.48 & 66.62 & 74.02 & 80.41 & $60.15(2.36)$ & $62.38(2.33)$ & $72.24(0.27)$ & $80.95(2.09)$ & AA \\
\hline & 60.59 & 63.97 & 71.90 & 78.77 & $55.19(0.79)$ & $55.19(0.76)$ & $68.26(0.49)$ & $77.68(2.24)$ & Kappa \\
\hline & 64.50 & 67.29 & 77.38 & 77.11 & $55.71(3.33)$ & $58.74(2.28)$ & $72.38(0.50)$ & $79.39(1.91)$ & $\mathrm{F} 1$ \\
\hline
\end{tabular}

- Random sampling (SVM,3DCNN): average and stddev of five generated training sets

- Cluster sampling (SVM): single run (deterministic sampler)

- Cluster sampling (3DCNN): average and stddev of five random seeds used for the weights initialization 


\section{Experimental Results}

\section{Indian Pines}

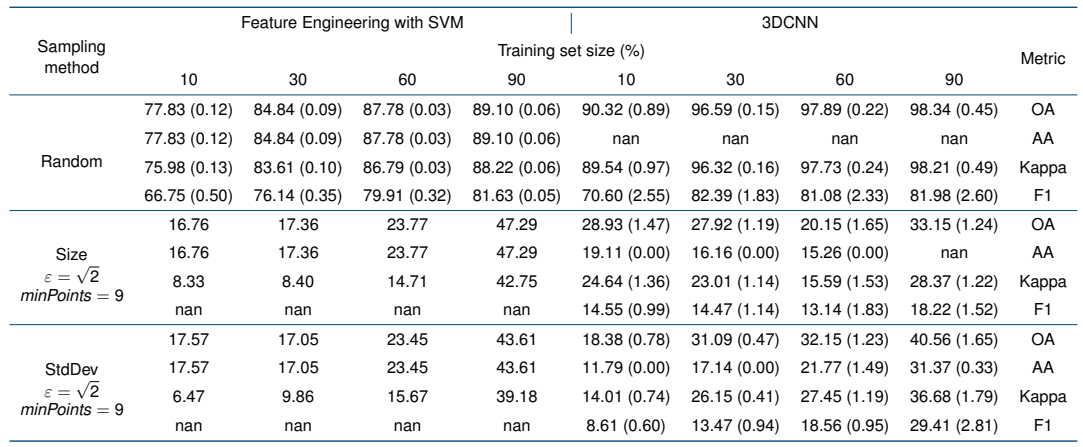

- Random sampling (SVM,3DCNN): average and stddev of five generated training sets

- Cluster sampling (SVM): single run (deterministic sampler)

- Cluster sampling (3DCNN): average and stddev of five random seeds used for the weights initialization 


\section{Random Sampling Evaluation}

Independence Assumption Violated

- The pattern recognition problem degrades to an almost memorization issue

- Classifying the same pixel class in the test set based on the previously seen similar instance in the training data is very likely

- Inherent to a variety of machine learning classifiers (SVMs, CNNs, etc.)

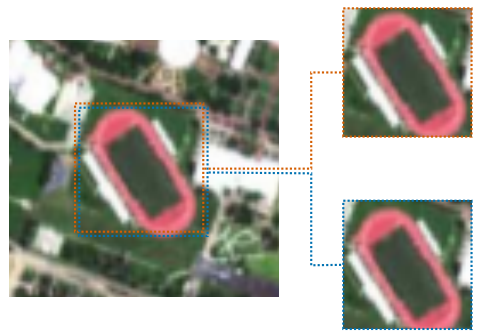

(3DCNN: insufficient offset between patches) 


\section{Overlap between train and test data}

\section{DCNN - Indian Pines Dataset}

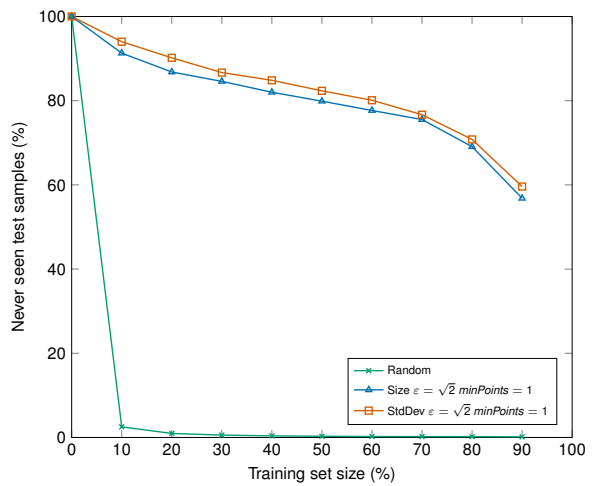




\section{Outline}

\section{Introduction}

\section{Proposed Cluster Sampling}

\section{Experimental Results}

Conclusions 


\section{Conclusions}

- Random sampling introduces systematic bias

- Issue for spectral-spatial classifiers (e.g., processing pipelines, CNNs, etc.)

- More dependence between train and test samples leads to higher accuracies

- Proposed controlled sampling approach based on DBSCAN clustering algorithm

- Easy definition of contiguous regions and train-test-set assignment prioritization

- Accuracies on unseen test data closer to an actual out-of-sample performance 


\section{The End}

\section{Thank you for your attention.}

\section{Code available at: https://github.com/Markus-Goetz/cluster-sampling}

This research has been supported in part by the SIMDAS project and in part by the DEEP-EST project, which have received funding from the European Union's Horizon 2020 research and innovation programme under the Grant Agreements No. 763558 and No. 754304, respectively.
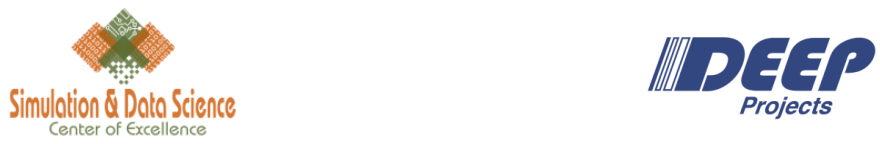
[1] J. Ball, D. Anderson, and C. S. Chan, "A Comprehensive Survey of Deep Learning in Remote Sensing: Theories, Tools and Challenges for the Community," SPIE Journal of Applied Remote Sensing (JARS), Special Section on Feature and Deep Learning in Remote Sensing Applications, Sep 2017.

[2] "Dataset: UC Merced Land Use - Computer vision lab."

http://weegee.vision. ucmerced.edu/datasets/landuse.html, 2010.

[3] "DataSet: WHU-RS19." http://www . xinhua-fluid.com/people/yangwen/WHU-RS19.html, 2012.

[4] "DataSet: RSSCN7." https ://www.dropbox . com/s/j80iv1a0mvhonsa/RSSCN7 . zip?dl=0, 2015.

[5] "DataSet: SAT-6 airborne." http://csc.lsu.edu/ saikat/deepsat/, 2015.

[6] "DataSet: Brazilian Coffee Scenes."

http://www . patreo.dcc . ufmg.br/2017/11/12/brazilian-coffee-scenes-dataset/, 2015.

[7] "DataSet: SIRI-WHU." http://www. Imars .whu.edu.cn/prof_web/zhongyanfei/e-code .html, 2016.

[8] "DataSet: NWPU-RESISC45." http://www .escience.cn/people/JunweiHan/NWPU-RESISC45.html, 2016.

[9] "DataSet: AID." http://www. Imars.whu.edu.cn/xia/AID-project.html, 2017.

[10] "DataSet: EuroSAT." http://madm.dfki.de/downloads, 2017.

[11] “DataSet: RSI-CB.” https ://github.com/lehaifeng/RSI-CB, 2017. 